\title{
DETERMINING THE PREVALENCE OF INV-POSITIVE AND AIL-POSITIVE YERSINIA ENTEROCOLITICA IN PIG TONSILS USING PCR AND CULTURE METHODS*
}

\author{
Milena Alicja Stachelska ${ }^{\bowtie}$ \\ Institute of Food Technology and Food Service, Lomza State University of Applied Sciences \\ Akademicka 14, 18-400 Łomża, Poland
}

\begin{abstract}
Background. Yersiniosis is believed to be the third most common intestinal zoonosis in the European Union, after campylobacteriosis and salmonellosis. Yersinia enterocolitica is the most common species responsible for human infections. Pigs are regarded as the biggest reservoir of pathogenic $Y$. enterocolitica strains, which are mainly isolated from pig tonsils. The aim of this paper is to examine the prevalence of $i n v$-positive and ail-positive $Y$. enterocolitica in pigs which were slaughtered in a Polish abattoir.

Material and methods. Real-time PCR and culture methods were used to assess the prevalence of pathogenic Y. enterocolitica strains in pig tonsils. Real-time PCR was applied to detect inv-positive and ail-positive Y. enterocolitica. $Y$. enterocolitica was also isolated by applying direct plating, unselective (tryptic soy broth) and selective (irgasan-ticarcillin-potassium chlorate bouillon) enrichment.

Results. A total of 180 pigs were studied, of which $85 \%$ and $32 \%$ respectively were found to be infected with inv-positive and ail-positive $Y$. enterocolitica. The $92 \mathrm{inv}$-positive and ail-positive isolates, from 57 culturepositive tonsils, underwent bio- and serotyping. The most common was bioserotype 4/O:3, which was found in $53(93 \%)$ out of 57 culture-positive tonsils. Strains of bioserotypes 2/O:5, 2/O:9 and 2/O:5.27 occurred in significantly lower numbers.

Conclusion. The prevalence of inv-positive and ail-positive $Y$. enterocolitica was found to be high in the tonsils of slaughtered pigs, using real-time PCR. The real-time PCR method for the detection and identification of pathogenic $Y$. enterocolitica is sensitive and specific, which has been verified by specificity and sensitivity tests using the pure cultures. Serotypes were distinguished from each other using PCR serotyping. The PCR method was essential in forming our conclusions.
\end{abstract}

Keywords: Yersinia enterocolitica, inv gene, ail gene, pig tonsils, biotyping, serotyping, the real-time PCR method, culture method

\section{INTRODUCTION}

Yersinia enterocolitica is a food-borne pathogen which can cause acute gastroenteritis and mesenteric lymphadenitis, which resembles appendicitis. Yersiniosis is thought to be the third most common intestinal zoonosis in the European Union, after campylobacteriosis and salmonellosis (Bucher et al., 2008; EFSA and

*This research was supported by funds granted by the Ministry of Science and Higher Education, within Project No BDS-15/ ITŻiG/10/2013.

『mstachelska@pwsip.edu.pl 
ECDC, 2016; Grahek-Ogden et al., 2007). Unfortunately, there is little epidemiological data concerning the assessment of pathogenic strains of $Y$. enterocolitica in animals in the European Union, as checking for this pathogen in animals is not obligatory in most countries (Fosse et al., 2008). Pig tonsils are known to be the biggest reservoir of pathogenic Y. enterocolitica strains (EFSA, 2011). The pathogenic strains have mostly been detected in pig tonsils, but they have also been isolated from environmental and food samples (Boyapalle et al., 2001; Fredriksson-Ahomaa and Korkeala, 2003; Lambertz et al., 2008). Moreover, there is a very strong resemblance between pig and human strains, which has been proven by DNA-based methods (Jacobsen et al., 2005).

However, there are some obstacles to isolating $Y$. enterocolitica strains from naturally contaminated tonsils or pork meat (Fredriksson-Ahomaa et al., 2000; Fredriksson-Ahomaa and Korkeala, 2003; Tauxe et al., 1987). Culture methods are insufficiently sensitive, which makes it impossible to differentiate pathogenic from non-pathogenic strains. What is more, it is essential to establish the pathogenicity of $Y$. enterocolitica strains, because most isolates from food and environmental samples are non-pathogenic. There are two genes which are chromosomally encoded and are responsible for the pathogenicity of $Y$. enterocolitica. They include the ail and inv genes. However, the inv gene can be present in both pathogenic and non-pathogenic strains. The ail gene determinates the pathogenicity of $Y$. enterocolitica strains. So the detection of only the $i n v$ gene is not sufficient for the detection of pathogenic strains. The detection of the ail gene is required to confirm the pathogenicity of $Y$. enterocolitica strains. The application of DNA-methods such as PCR techniques enables us to detect inv-positive and ail-positive $Y$. enterocolitica in natural samples, achieving a very high level of sensitivity and specificity (Zheng et al., 2008).

On the other hand, to achieve epidemiological data, the culture method should be carried out in combination with the PCR method to differentiate between $Y$. enterocolitica isolates. It is necessary to apply selective enrichment, especially for the isolation of $Y$. enterocolitica strains from food samples. There is no single procedure which is commercially available to collect all bioserotypes. The most frequently applied selective broth is the irgasan-ticarcillin-potassium chlorate (ITC) broth, which is used especially for the detection of bioserotype 4/O:3 (Fredriksson-Ahomaa et al., 2001a; Lambertz et al., 2007).

Bio- and serotyping have been widely applied to differentiate isolates. The biotyping scheme was suggested by Wauters et al. (1987) and universally accepted. Pathogenic isolates, including biotypes 1B, $2-5$ can be discriminated from biotype $1 \mathrm{~A}$ by using the pyrazinamidase test. Serotypes such as O:3, O:5 and $\mathrm{O}: 8$, which are responsible for human diseases, can also be met in non-pathogenic $Y$. enterocolitica strains. This means that a very precise biochemical characterisation for the correct identification of the strains is required, before and after serological typing, for every related species and biotype 1A strain which is present in food and environmental samples. Additionally, the subtyping of $Y$. enterocolitica strains which appear among the same bioserotype is a necessity in order to assess the animal reservoir or to estimate the disease outbreaks (Galindo et al., 2011).

The aims of this study were (1) to assess the prevalence of inv-positive and ail-positive $Y$. enterocolitica in pig tonsils at a slaughterhouse in Podlaskie voivodeship in Poland, using real-time PCR and culture methods, and (2) consequently to bio- and serotype the isolates to achieve more epidemiological information on Y. enterocolitica strains at the slaughterhouse.

\section{MATERIAL AND METHODS}

\section{Sampling and sample preparation}

Tonsils from 180 slaughtered pigs were taken from a slaughterhouse in Podlaskie voivodeship in Poland, in January and February 2017. The pigs were transported to the slaughterhouse from 12 different farms in the eastern region of Poland. Tonsils were removed aseptically immediately after evisceration, placed into sterile plastic bags and delivered to the laboratory under chilled conditions. Tonsil samples were aseptically cut into small pieces, and $10 \mathrm{~g}$ of tonsils were put into a sterile stomacher bag. Samples were homogenized with $90 \mathrm{ml}$ of tryptic soy broth (Oxoid, UK) for $2 \mathrm{~min}$ in a stomacher (Colworth Stomacher 400, Seward Ltd, London, UK). This homogenate was used for the PCR and culture method investigations. 


\section{Detection of inv-positive and ail-positive} Y. enterocolitica using the real-time PCR method Real-time PCR was applied to detect inv-positive and ail-positive $Y$. enterocolitica after $18 \mathrm{~h}$-enrichment in tryptic soy broth at $30^{\circ} \mathrm{C}$. DNA from a culture grown in trypton soy broth was taken for purification, for real-time PCR analysis. $1 \mathrm{ml}$ of enrichment culture was pipetted into a $2 \mathrm{ml}$ microcentrifuge screw-cap tube and was centrifuged at $13000 \times \mathrm{g}$ for $5 \mathrm{~min}$. Then the supernatant was discarded using a pipet. Care was taken not to disrupt the pellet. Then $200 \mu 1$ of Fast Lysis Buffer (Syngen Biotech, Germany) was added to the bacterial pellet, the tube was tightly capped and the pellet was resuspended by vigorous vortexing. Then the microcentrifuge tube was placed into a thermal shaker $(800 \mathrm{rpm})$ set to $100^{\circ} \mathrm{C}$. The sample was heated for $10 \mathrm{~min}$. The sample was removed and cooled to room temperature $\left(15-25^{\circ} \mathrm{C}\right)$ for $2 \mathrm{~min}$. The tube was centrifuged at $13000 \times \mathrm{g}$ for $5 \mathrm{~min}$. Then $100 \mu \mathrm{l}$ of the supernatant was transferred to a fresh $1.5 \mathrm{ml}$ microcentrifuge tube. This supernatant was subjected to further investigation by real-time PCR (Rotor Mx3000P Stratagene Agilent Technologies, USA), using PowerChek $^{\mathrm{TM}}$ Yersinia enterocolitica Real-time PCR Kit Protocol. The kit was used to detect the specific sequence of inv- and ail-genes for $Y$. enterocolitica. This kit contains a Real-time PCR MasterMix, with enzyme components and the specific primer/probe set for the test by Real-time assay and for the Internal Control (IC) system for the reliability of results. This is a qualitative Duplex Real-time PCR test which includes the amplification of pathogen specific genes (inv, ail) and the Internal Control (IC) with specific primers and probes labelled with fluorescent dyes.

Table 1. PCR reaction mixture

\begin{tabular}{lc}
\hline \multicolumn{1}{c}{ Composition } & Volume, $\mu 1$ \\
\hline Primer/Probe Mix & 4 \\
2X Real-time PCR Master Mix & 10 \\
Template DNA (Control DNA or DNA isolated & 5 \\
from food-borne pathogens) & \\
TE buffer (PCR water, negative control) & 1 \\
Total & 20 \\
\hline
\end{tabular}

Table 2. The thermal profiles

\begin{tabular}{ccc}
\hline Temperature, ${ }^{\circ} \mathrm{C}$ & Time & Cycle \\
\hline 95 & $10 \mathrm{~min}$ & 1 \\
95 & $15 \mathrm{~s}$ & 40 \\
$60^{*}$ & $1 \mathrm{~min}$ & \\
\hline
\end{tabular}

${ }^{*}$ Detection of the fluorescence at this step.

The total reaction volume was $20 \mu \mathrm{l}$, the volume of DNA was $5 \mu 1$. The reaction mixture was prepared according to the Table 1. The Real-time PCR conditions are presented in Table 2 .

\section{Detection of inv-positive and ail-positive \\ $Y$. enterocolitica using the culture method}

$Y$. enterocolitica was isolated by applying direct plating, unselective and selective enrichment. $100 \mu 1$ of the homogenate was plated on selective CIN (Yersinia Selective Agar Base and Yersinia Selective Supplement, Oxoid, UK) agar plates by a spiral plate machine (Eddie Jet, IUL Instruments, Barcelona, Spain). The homogenate was also enriched in unselective tryptic soy broth (Oxoid, UK) at $30^{\circ} \mathrm{C}$ for $18 \mathrm{~h}$ and subsequently $10 \mu \mathrm{l}$ of $18 \mathrm{~h}$-enrichment was streaked onto CIN agar plates. Another selective enrichment was applied. $1 \mathrm{ml}$ of the homogenate was added to $9 \mathrm{ml}$ of irgasan-ticarcillin-potassium chlorate bouillon (ITC, Oxoid, UK), then incubated at $30^{\circ} \mathrm{C}$ for $18 \mathrm{~h}$ and then $10 \mu \mathrm{l}$ from the selective enrichment was streaked onto a CIN agar plate. All CIN agar plates were incubated at $30^{\circ} \mathrm{C}$ for $18 \mathrm{~h}$. Suspected colonies resembling a "bull's eye" were checked for urea hydrolyse. Urea-positive isolates were identified with API 20E. The inv-positive Y. enterocolitica strains were confirmed by the real-time PCR method.

\section{Biotyping and serotyping of inv-positive and ail-positive $Y$. enterocolitica isolates}

92 inv-positive and ail-positive $Y$. enterocolitica isolates coming from 57 culture-positive tonsils were bioand serotyped. The biotype was assessed by pyrazinamidase and tween activities, the ability to hydrolyse esculin, the ability to produce indole, and the the ability to ferment salicin, xylose and trehalose (Wauters et al., 1987). Serotyping was performed with slide agglutination with commercially available $Y$. enterocolitica 
O:3, O:5, O:9 and O:27 (Argenta, Poland). Serotyping was also carried out by real-time PCR using a primer/ probe set. Serotypes O:3 and O:9 were identified by the wbbU (Skurnik, 1999) and per (Jacobsen et al., 2005) genes respectively.

\section{Assessment of specificity of the assay using the pure cultures}

Specificity of the assay was checked by using 16 bacterial strains (Table 3). Pure cultures of Yersinia spp. were cultivated in trypton soy broth (TSB) broth at $30^{\circ} \mathrm{C}$ for $16-18 \mathrm{~h}$. Bacterial DNA was extracted from $1 \mu \mathrm{l}$ of overnight enrichment, which was transferred to $100 \mu 1$ sterile distilled water and subsequently boiled for 10 min to lyse the bacteria. Two $\mu$ l of the boiled bacteria suspension was used as template in PCR.

\section{RESULTS AND DISCUSSION}

In the study, pig tonsil samples were investigated, as they constitute the biggest reservoir of pathogenic $Y$. enterocolitica strains. Y. enterocolitica strains are found in numbers 10 times higher in pig tonsils than in pig faeces after slaughter. It means that tonsils are a more important source of carcass contamination than faeces in the slaughterhouse (Laukkanen-Ninios et al., 2014).

The prevalence of inv-positive and ail-positive $Y$. enterocolitica isolates present in tonsils coming from slaughtered pigs was found to be very high using real-time PCR. The prevalence rate was found to be $85 \%$ with a set of probe/primers (Table 4 ). The specificity and sensitivity test for the detection of $i n v-$ -positive and ail-positive $Y$. enterocolitica was carried out on 16 strains. The assay was found to be specific for all potential pathogenic strains of $Y$. enterocolitica (Table 3).

This method has been used in many studies assessing the prevalence of pathogenic $Y$. enterocoliti$c a$ strains in pig tonsils. The results of these studies showed that the detection rate seemed to be higher with the real-time PCR compared to culture methods (Boyapalle et al., 2001; Stenkova et al., 2008; Vázlerová and Steinhauserová, 2006). The real-time PCR assay was $1000-10,000$ times more specific and more

Table 3. The specificity and sensitivity test using 16 strains

\begin{tabular}{|c|c|c|}
\hline Species of Yersinia & Original strain no. & The real-time PCR \\
\hline Y. enterocolitica (pathogenic bioserotype) & YE1 & positive \\
\hline Y. enterocolitica (pathogenic bioserotype) & YE3 & positive \\
\hline Y. enterocolitica (pathogenic bioserotype) & YE5 & positive \\
\hline Y. enterocolitica (pathogenic bioserotype) & YE7 & positive \\
\hline Y. enterocolitica (pathogenic bioserotype) & YE165 & positive \\
\hline Y. enterocolitica (pathogenic bioserotype) & KNG22703 & positive \\
\hline Y. enterocolitica (pathogenic bioserotype) & $2516-87$ & positive \\
\hline Y. enterocolitica (pathogenic bioserotype) & 8081 & positive \\
\hline Y. enterocolitica (pathogenic bioserotype) & WA & positive \\
\hline Y. enterocolitica (pathogenic bioserotype) & W22703 & positive \\
\hline Y. enterocolitica subsp. palearctica (pathogenic bioserotype) & $105.5 \mathrm{R}(\mathrm{r})$ & positive \\
\hline Y. enterocolitica subsp. palearctica (pathogenic bioserotype) & Y11 & positive \\
\hline Y. enterocolitica subsp. palearctica (pathogenic bioserotype) & 8081 & positive \\
\hline Y. enterocolitica (nonpathogenic bioserotype) & HYE5283 & negative \\
\hline Y. enterocolitica (nonpathogenic bioserotype) & HYE9180 & negative \\
\hline Y. enterocolitica (nonpathogenic bioserotype) & $\mathrm{T} 16.1 \mathrm{M}$ & negative \\
\hline
\end{tabular}


Table 4. Frequency of inv-positive and ail-positive $Y$. enterocolitica strains in 180 pig tonsil samples from pigs coming from 12 farms with various detection methods

\begin{tabular}{lcc}
\hline \multicolumn{1}{c}{ Method } & $\begin{array}{c}\text { Number } \\
\text { of positive } \\
\text { samples }\end{array}$ & Percentage \\
\hline $\begin{array}{l}\text { The real-time PCR with a set of } \\
\text { probe/primers }\end{array}$ & 153 & 85 \\
Culture method & 57 & 32 \\
$\begin{array}{l}\text { Direct plating on CIN agar } \\
\begin{array}{l}\text { Overnight enrichment in tryptic } \\
\text { soy broth (non selective broth) } \\
\text { and plating on CIN agar }\end{array}\end{array}$ & 42 & 23 \\
$\begin{array}{l}\text { Selective enrichment in ITC } \\
\text { (irgasan-ticarcillin-potassium } \\
\text { chlorate) broth and plating } \\
\text { on CIN agar }\end{array}$ & 42 & 21 \\
\hline
\end{tabular}

reliable than the culture method or the traditional PCR assay (Boyapalle et al., 2001; Messelhäusser et al., 2011).

However, as the inv-gene can be present in both pathogenic and non-pathogenic strains, the confirmation of the pathogenicity of $Y$. enterocolitica was carried out by the detection other genes present in pathogenic strains, mostly the ail-gene. Momtaz et al. (2013) used other genes to detect pathogenic $Y$. enterocolitica strains. Among these were chromosomal virulence genes such as inv, ail and yst A, and the plasmid-encoded virulence factors such as yadA and virF. They used multiplex PCR assay and detected the chromosomal virulence genes, including inv $(100 \%)$, ail $(95.65 \%)$, and ystA $(93.47 \%)$, and plasmid-encoded virulence factors including $\operatorname{yad} A(82.60 \%)$ and virF $(58.69 \%)$, out of the total of $46 Y$. enterocolitica serotype O:3 strains. The results of their studies were very similar to those carried out by Cocolin and Comi (2005) who isolated the 6 biotypes and 50 serotypes of $Y$. enterocolitica and proved that incidences of $Y$. enterocolitica serotype 0:5.27 and biotype 3 were highest in chicken meat.

Momtaz et al. (2013) used both molecular and culture methods to characterize $Y$. enterocolitica isolates in chicken meat. Results indicated that 132 of the 720 $(18.3 \%)$ chicken meat samples studied were infected with $Y$. enterocolitica. Out of 132 samples positive for $Y$. enterocolitica, the prevalence of the 6 biotypes were found to be; $1 \mathrm{~A}(0 \%), 1 \mathrm{~B}(0 \%), 2(18.18 \%)$, $3(52.27 \%), 4(17.42 \%)$, and $5(12.12 \%)$, and the most prevalent serotypes were O:3 (34.84\%), O:5.27 (59.84\%), O:8 (5.30\%), and O:9 (0\%). Another study carried out by Fredriksson-Ahomaa and Korkeala (2003) showed that pigs are a major reservoir of pathogenic $Y$. enterocolitica of bioserotype 4/O:3, the most common type found in humans. However, Momtaz et al. (2013) indicated that that O:5.27/2 was the most frequent serobiotype of $Y$. enterocolitica isolated from chicken meat samples. Their study confirmed that the O:5.27/2 serobiotype of $Y$. enterocolitica caused the most human infections through consumption of contaminated chicken meat. They also confirmed that $y s t A$, with an incidence of $93.47 \%$, was one of the most prevalent virulence genes of the $Y$. enterocolitica that was isolated from chicken meat samples. Another study conducted by Favier et al. (2005) indicated that $38.65 \%$ of chicken meat samples were contaminated with $Y$. enterocolitica, which was higher than the results of Momtaz et al. (2013) (18.33\%). All $Y$. enterocolitica 2/O:9 strains gave results related to virulence by phenotypic tests, and exhibited the genotype $v i r F^{+} m y f A^{+}$ail $l^{+} y s t A^{+}$, whereas biotype $1 \mathrm{~A}$ strains showed the genotype virF-myfA-ail ${ }^{+} \mathrm{ystA}^{+} \mathrm{ystB}^{+}$. The study carried out by Zheng et al. (2008) indicated that the prevalence of virulence genes tested in $160 \mathrm{Y}$. enterocolitica isolates were inv (100\%), ail (94\%), ystA (93\%), ystB (7.5\%), ystC (5\%), yadA (89\%), and virF $(82 \%)$ in patients with diarrhoea.

A study conducted by Lambertz and Danielsson-Tham (2005) proved that pigs were the main reservoir of food-borne pathogenic $Y$. enterocolitica. The researchers combined culturing and PCR methods for detection, and a multiplex PCR was developed for the detection of virulence genes (yst, $r f b C$, ail, virF). In all, 118 pork products ( 91 raw and 27 ready-to-eat) were collected. Pathogenic Yersinia spp. were detected by PCR in $9.89 \%$ ( 9 out of 91 ) of the raw pork samples but in none of the ready-to-eat products.

In our study, the final isolation rate of pathogenic $Y$. enterocolitica strains detected in pig tonsils was found to be $32 \%$ after direct plating, overnight enrichment and selective enrichment (Table 4). It was found that at least $10^{6} \mathrm{cfu} / \mathrm{g}$ of pathogenic $Y$. enterocolitica 
Stachelska, M. A. (2017). Determining the prevalence of inv-positive and ail-positive Yersinia enterocolitica in pig tonsils using PCR and culture methods. Acta Sci. Pol. Technol. Aliment., 16(3), 303-310. http://dx.doi.org/10.17306/J.AFS.2017.0495

Table 5. Types of biotypes and serotypes of $Y$. enterocolitica isolated from culture-positive tonsils

\begin{tabular}{|c|c|c|c|c|c|c|c|c|c|}
\hline \multirow{2}{*}{ Species } & \multirow{2}{*}{ Biotype } & \multirow{2}{*}{ Serotype } & \multicolumn{5}{|c|}{ Results of PCR that targets } & \multirow{2}{*}{ Number of isolates } & \multirow{2}{*}{$\begin{array}{l}\text { Number of culture- } \\
\text { positive tonsils }\end{array}$} \\
\hline & & & inv & ail & $r f b \mathrm{C}$ & per & $w b b U$ & & \\
\hline Y. enterocolitica & 4 & $0: 3$ & + & + & - & - & + & $75 / 92(82 \%)$ & $53 / 57(93 \%)$ \\
\hline Y. enterocolitica & 2 & O:5 & + & + & - & - & - & $8 / 92(9 \%)$ & $7 / 57(12 \%)$ \\
\hline Y. enterocolitica & 2 & O:9 & + & + & - & + & - & $3 / 92(3 \%)$ & $4 / 57(7 \%)$ \\
\hline Y. enterocolitica & 2 & O:5.27 & + & + & - & - & - & $6 / 92(7 \%)$ & $3 / 57(5 \%)$ \\
\hline
\end{tabular}

strains are required to be detected in food. Selective enrichment was found to be more productive and to give a higher number of $Y$. enterocolitica cells in comparison to non-selective enrichment, which was demonstrated in this study (Aldová et al., 1990). It should be noted that selective enrichment enabled us primarily to recover isolates belonging to bioserotype 4/O:3 (Fredriksson-Ahomaa and Korkeala, 2003). It was also confirmed that ITC broth was designed for the detection and isolation of serotypes $\mathrm{O}: 3$ and $\mathrm{O}: 9$, but was not optimal for isolation of serotype $0: 9$. The lowest prevalence of pathogenic $Y$. enterocolitica strains was observed when they were cultivated in non-selective enrichment broth, compared to the high prevalence rates found using the PCR method.

A total of 180 pigs investigated, of which $85 \%$ and $32 \%$ respectively were found to be infected with pathogenic strains, using PCR and culture methods. The 92 inv-positive and ail-positive isolates from 57 culture-positive tonsils were bio- and serotyped. The most frequent bioserotype was found to be $4 / \mathrm{O}: 3$, isolated from $93 \%(53 / 57)$ of the culture-positive tonsils. Moreover, tonsils also contained inv-positive and ail-positive $Y$. enterocolitica bioserotypes such as 2/O:5 and $2 / 0: 9$, which were isolated from $12 \%(7 / 57)$ and $7 \%(4 / 57)$ of the culture-positive tonsils (Table 4$)$.

Among 92 inv-positive and ail-positive $Y$. enterocolitica isolates coming from 57 culture-positive tonsils, there were a few isolates belonging to biotype 4 which did not agglutinate with $\mathrm{O}: 3, \mathrm{O}: 5$ or $\mathrm{O}: 9$ antisera or agglutinated very poorly with $\mathrm{O}: 3$ antiserum and all six isolates of serotype 0:5.27 did not visibly agglutinate with O:5. The next step was to carry out serotyping by PCR. All inv-positive and ail-positive $Y$. enterocolitica isolates which belonged to biotype
4 were $w b b U$ positive and per negative, proving that these isolates belonged to serotype O:3. Three of the nine isolates belonging to biotype 2 were per positive and $r f b \mathrm{C}$ negative, confirming that these three isolates belonged to serotype O:9. The six isolates which belonged to biotype 2, which were both $r f b \mathrm{C}$ and per negative, agglutinated well with $\mathrm{O}: 27$ antiserum and belonged to serotype 0:5.27 (Table 5).

The most common bioserotype was 4/O:3, which occurred in $53(93 \%)$ out of 57 culture-positive tonsils. This bioserotype 4/O:3 commonly occurs in tonsils and is frequently isolated from slaughtered pigs (Fredriksson-Ahomaa et al., 2001b). It is responsible for human yersiniosis in most European countries. Isolates belonging to biotype 2 were also isolated from Polish pig tonsils. Biotype 2 was very rarely found in pigs. It is known to be common in ruminants (Table 5).

\section{CONCLUSION}

The prevalence of $i n v$-positive and ail-positive $Y$. enterocolitica was found to be high in the tonsils of slaughtered pigs using real-time PCR. The real-time PCR method for the detection and identification of pathogenic $Y$. enterocolitica was found to be rapid and accurate. This method is highly sensitive and specific, which was confirmed by carrying out the specificity and sensitivity test for the detection of inv-positive and ail-positive $Y$. enterocolitica strains. The most common was bioserotype 4/O:3 which occurred in 53 (93\%) out of 57 culture-positive tonsils. Strains of bioserotype 2/O:5, bioserotypes 2/O:9 and 2/O:5.27 occurred in significantly lower numbers. Serotypes were distinguished from each other by PCR serotyping. The PCR method was found to be essential in making conclusions. 


\section{REFERENCES}

Aldová, E., Švandová, E., Votýpka, J., Šourek, J. (1990). Comparative study of culture methods to detect Yersinia enterocolitica serogroup $\mathrm{O} 3$ on swine tongues. Zentralbl. Bakteriol., 272, 3, 306-312.

Boyapalle, S., Wesley, I. V., Hurd, H. S., Reddy, P. G. (2001). Comparison of culture, multiplex, and 5' nuclease polymerase chain reaction assays for the rapid detection of Yersinia enterocolitica in swine and pork products. J. Food Prot., 64, 9, 306-312.

Bucher, M., Meyer, C., Grötzbach, B., Wacheck, S., Stolle, A., Fredriksson-Ahomaa, M. (2008). Epidemiological data on pathogenic Yersinia enterocolitica in Southern Germany during 2000-2006. Foodborne Pathog. Dis., 5, $3,273-280$.

Cocolin, L., Comi, G. (2005). Use of a culture-independent molecular method to study the ecology of Yersinia spp. in food. Int. J. Food Microbiol., 105, 71-82.

EFSA (2011). EFSA panels on biological hazards (BIOHAZ), on contaminants in the food chain (CONTAM), and on animal health and welfare (AHAW). Scientific opinion on the public health hazards to be covered by inspection of meat (swine). EFSA J., 9, 10, 198.

EFSA and ECDC (2016). The European Union summary report on trends and sources of zoonoses, zoonotic agents and food-borne outbreaks in 2014. EFSA J., 14(12), 4634.

Favier, G. I., Escudero, M. E., de Guzmán, A. M. (2005). Genotypic and phenotypic characteristics of Yersinia enterocolitica isolated from the surface of chicken eggshells obtained in Argentina. J. Food Prot., 68, 1812-1815.

Fosse, J., Seegers, H., Magras, C. (2008). Foodborne zoonoses due to meat: a quantitative approach for a comparative risk assessment applied to pig slaughtering in Europe. Vet. Res., 39, 1, 1-16.

Fredriksson-Ahomaa, M., Korte, T., Korkeala, H. (2000). Contamination of carcasses, offals, and the environment with yadA-positive Yersinia enterocolitica in a pig slaughterhouse. J. Food Prot., 63, 1, 31-35.

Fredriksson-Ahomaa, M., Hallanvuo, S., Korte, T., Siitonen, A., Korkeala, H. (2001a). Correspondence of genotypes of sporadic Yersinia enterocolitica bioserotype 4/O:3 strains from human and porcine sources. Epidemiol. Infect., 127, 1, 37-47.

Fredriksson-Ahomaa, M., Bucher, M., Hank, C., Stolle, A., Korkeala, H. (2001b). High prevalence of Yersinia enterocolitica 4:O3 on pig offal in southern Germany:
A slaughtering technique problem. Syst. Appl. Microbiol., 24, 3, 457-463.

Fredriksson-Ahomaa, M., Korkeala, H. (2003). Low occurrence of pathogenic Yersinia enterocolitica in clinical, food, and environmental samples: a methodological problem. Clin. Microbiol. Rev., 16, 2, 220-229.

Galindo, C. L., Rosenzweig, J. A., Kirtley, M. L., Chopra, A. K. (2011). Pathogenesis of $Y$. enterocolitica and $Y$. pseudotuberculosis in human yersiniosis. J. Pathog., 17, $1-16$.

Grahek-Ogden, D., Schimmer, B., Cudjoe, K. S., Nygard, K., Kapperud, G. (2007). Outbreak of Yersinia enterocolitica serogroup 0:9 infection and processed pork, Norway. Emerg. Infec. Dis., 13, 5, 754-756.

Jacobsen, N. R., Bogdanovich, T., Skurnik, M., Lubeck, P. S., Ahrens, P., Hoorfar, J. (2005). A real-time PCR assay for the specific identification of serotype O:9 of Yersinia enterocolitica. J. Microbiol. Meth., 63, 151-156.

Lambertz, S. T., Danielsson-Tham, M. L. (2005). Identification and characterization of pathogenic Yersinia enterocolitica isolates by PCR and pulsed-field gel electrophoresis. Appl. Environ. Microbiol., 71, 3674-3681.

Lambertz, S. T., Granath, K., Fredriksson-Ahomaa, M., Johansson, K. E., Danielsson-Tham, M. L. (2007). Evaluation of a combined culture and PCR method (NMKL-163A) for detection of presumptive pathogenic Yersinia enterocolitica in pork products. J. Food Protec., 70, 2, 335-340.

Lambertz, S. T., Nilsson, C., Hallanvuo, S., Lindblad, M. (2008). Real-time PCR method for detection of pathogenic Yersinia enterocolitica in food. Appl. Environ. Microbiol., 74, 19, 6060-6067.

Laukkanen-Ninios, R., Fredriksson-Ahomaa, M., Maijala, R., Korkeala, H. (2014). High prevalence of pathogenic Yersinia enterocolitica in pig cheeks. Food Microbiol., 43, 50-52.

Messelhäusser, U., Kämpf, P., Colditz, J., Bauer, H., Schreiner, H., Höller, C., Busch, U. (2011). Qualitative and quantitative detection of human pathogenic Yersinia enterocolitica in different food matrices at retail level in Bavaria. Foodborne Pathog. Dis., 8, 1, 39-44.

Momtaz, H., Rahimian, M. D., Dehkordi, S. F. (2013). Identification and characterization of Yersinia enterocolitica isolated from raw chicken meat based on molecular and biological techniques. J. Appl. Poult. Res., 22, 137-145.

Skurnik, M. (1999). Molecular genetics of Yersinia lipopolysaccharide. In J. B. Goldberg (Ed.), Genetics of bacterial polysaccharides (pp. 23-52). Boca Raton, New York: CRC Press. 
Stenkova, A. M., Isaeva, M. P., Rasskazov, V. A. (2008). Development of a multiplex PCR procedure for detection of Yersinia genus with identification of pathogenic species (Y. pestis, Y. pseudotuberculosis, and Y. enterocolitica). Molec. Genet. Microbiol. Virol., 23, 3, 119-125.

Tauxe, R. V., Vandepitte, J., Wauters, G., Martin, S. M., Goossens, V., De Mol, P., ..., Thiers, G. (1987). Yersinia enterocolitica infections and pork. Lancet, 1, 8542, 1129-1132.

Vázlerová, M., Steinhauserová, I. (2006). The comparison of the methods for the identification of pathogenic serotypes and biotypes of Yersinia enterocolitica: Microbiological methods and PCR. Czech J. Food Sci., 24, 5, 217-222.
Wauters, G., Kandolo, K., Janssens, M. (1987). Revised biogrouping scheme of Yersinia enterocolitica. Contrib. Microbiol. Immunol., 9, 14-21.

Weynants, V., Jadot, V., Denoel, P., Tibor, A., Letesson, J. J. (1996). Detection of Yersinia enterocolitica serogroup O:3 by a PCR method. J. Clin. Microbiol., 34, $1224-1227$.

Zheng, H., Sun, Y., Mao, Z., Jiang, B. (2008). Investigation of virulence genes in clinical isolates of Yersinia enterocolitica. FEMS Immunol. Med. Microbiol., 53, 368-374. 